\title{
Development of magnetism in strongly correlated cerium systems: Non-Kondo mechanism for moment collapse
}

\author{
Eric M. Collins, Nicholas Kioussis, and Say Peng Lim \\ Department of Physics and Astronomy, California State University Northridge, Northridge, California 91330-8268 \\ Bernard R. Cooper \\ Department of Physics, West Virginia University, Morgantown, West Virginia 26506-6315
}

(Received 3 August 2000)

\begin{abstract}
We present an $a b$ initio-based method which gives clear insight into the interplay between the band- $f$ hybridization, the band- $f$ Coulomb exchange, and the crystal-field interactions, as the degree of $4 f$ localization is varied across a series of strongly correlated cerium systems. The predictions for the ordered magnetic moments, magnetic structure, and ordering temperatures are in excellent agreement with experiment, including the occurrence of a moment collapse of non-Kondo origin. In contrast, spin- and orbitally polarized $a b$ initio density functional calculations and local density approximation $+U$ calculations fail to predict, even qualitatively, the trend of the unusual magnetic properties.
\end{abstract}

Treating the consequences of correlation effects on magnetic behavior in the transitional (neither atomiclike nor itinerant) $4 f$ or $5 f$ localization regime, provides one of the central problems of condensed matter physics. ${ }^{1,2}$ There is an extremely interesting range of phenomena, but with great difficulty in treating the theory of these phenomena adequately, especially in a way providing the ability to predict the behavior of specific materials. ${ }^{1,2}$ An adequate treatment requires treating the interelectronic Coulomb interaction, i.e., the correlation effects, as constrained by exchange symmetry. ${ }^{3-5}$ In this paper, we demonstrate an approach for treating these difficulties by predicting the interesting and complex behavior of an important series of cerium compounds.

The isostructural (rock-salt structure) series of the cerium monopnictides $\mathrm{Ce} X \quad(X=\mathrm{P}, \mathrm{As}, \mathrm{Sb}, \mathrm{Bi})$ and monochalcogenides $(X=\mathrm{S}, \mathrm{Se}, \mathrm{Te})$ have become prototype model systems for study, because of their unusual magnetic properties. ${ }^{6-12}$ These strongly correlated electron systems offer the opportunity to vary systematically the chemical environment and cerium-cerium separation on going down the pnictogen or chalcogen column, and hence tailor the degree of $4 f$ localization from the weakly correlated limit in the lighter systems to the strongly correlated limit in the heavier systems. ${ }^{6-12}$ The calculated single-impurity Kondo temperature, presented below, is much smaller than the magnetic ordering temperature; hence this series lies in the magnetic regime of the Kondo phase diagram. ${ }^{13}$ Nevertheless, we demonstrate that the sensitivity of the band- $f$ hybridization, band- $f$ Coulomb exchange, and crystal-field (CF) interactions to chemical environment gives rise to a variety of interesting unusual magnetic properties, in agreement with experiment across the series, including the occurrence of a non-Kondo moment collapse.

Experimentally, the large magnetic anisotropy changes from favoring $\langle 001\rangle$ alignment in the pnictides to $\langle 111\rangle$ in the chalcogenides. The low-temperature ordered magnetic moment increases with increasing lattice constant for the pnic- tides from $0.80 \mu_{B}$ in $\mathrm{CeP}$ to $2.1 \mu_{B}$ in $\mathrm{CeSb}$ and $\mathrm{CeBi}$, ${ }^{6,7}$ while it decreases with increasing lattice constant for the chalcogenides from $0.57 \mu_{B}$ in $\mathrm{CeS}$ to $0.3 \mu_{B}$ in CeTe. ${ }^{6-8}$ The magnetic moment collapse from $\mathrm{CeSb}$ to $\mathrm{CeTe}$, with both systems having about the same lattice constant, is indicative of the sensitivity of the magnetic interactions to chemical environment. The ordering temperature increases from $8 \mathrm{~K}$ in $\mathrm{CeP}$ to $26 \mathrm{~K}$ in $\mathrm{CeBi}$ for the pnictides, whereas it decreases from $8.4 \mathrm{~K}$ in $\mathrm{CeS}$ to $2.2 \mathrm{~K}$ in CeTe. ${ }^{6-12,14}$ Another unusual feature is the large suppression of the $\mathrm{CF}$ splitting $\Delta_{\mathrm{CF}}$ of the $\mathrm{Ce}^{3+} 4 f_{5 / 2}$ multiplet between the $\Gamma_{7}$-doublet $\mathrm{CF}$ ground state and the excited $\Gamma_{8}$ quartet, from values expected from the behavior of the heavier isostructural rareearth pnictides or chalcogenides. ${ }^{15,16}$ This can be understood $^{17}$ as arising from band- $f$ hybridization effects.

In this paper we present material-predictive results from two methods, one ab initio based and one $a b$ initio, to study the change of magnetic properties across this series. The first, $a b$ initio-based method employs the degenerate Anderson lattice model which explicitly incorporates the hybridization and the Coulomb exchange interactions on an equal footing. ${ }^{4}$ This method gives clear insight into the role of the three pertinent interactions: (1) The band- $f$ hybridizationinduced intercerium magnetic coupling, (2) the corresponding effects of band- $f$ Coulomb exchange, and (3) the CF interaction. This approach allows us to understand the interplay between these interactions as the degree of $4 f$ localization is varied across the series. It predicts magnetic moments, magnetic structures, and ordering temperatures in excellent agreement with experiment. Thus, this approach allows to understand and predict a number of key features of observed behavior. First, is the very low moment and low ordering temperature of the antiferromagnetism observed in CeTe, an incipient heavy Fermion system. ${ }^{18}$ We predict the magnetic moment and ordering temperature collapse from $\mathrm{CeSb}$ to CeTe, both systems having about the same lattice constant but CeTe having an additional $p$ electron. The earlier work of Sheng and Cooper ${ }^{4}$ showed that this magnetic ordering re- 
duction is accurately predicted without including any CF effects. An erroneous statement appears in the recent review article by Santini et al. ${ }^{19}$ stating that CF effects played an important role in the calculated results of Sheng and Cooper. ${ }^{4}$ This is incorrect, since CF effects were not included in these calculations. We show in this paper that including the CF effects modifies this behavior only quantitatively. Thus, the origin of the moment collapse is neither of Kondo origin or CF effect. Second, our results demonstrate the great sensitivity of the relative importance of hybridization and Coulomb exchange effects on magnetic ordering depending on the degree of $4 f$ localization. The band- $f$ Coulomb exchange mediated interatomic $4 f-4 f$ interactions are more important for the magnetic behavior of the heavier, more localized, systems, whereas the hybridization-mediated coupling is more important for the lighter, more delocalized systems. Third, we show that for the lighter more delocalized systems the CF interactions are much larger than the intercerium interactions and hence dominate the magnetic behavior. Finally, we predict the experimentally observed change of the ground-state magnetic structure from the $\langle 001\rangle \mathrm{AF}-\mathrm{IA}$ $(\uparrow \uparrow \downarrow \downarrow)$ in $\mathrm{CeBi}$ and $\mathrm{CeSb}$ to the $\langle 001\rangle \mathrm{AF}-I(\uparrow \downarrow)$ in CeAs and CeP. In sharp contrast, the second, ab initio method, based on density functional theory within the local density approximation (LDA), ${ }^{20,21}$ and on the LDA $+U$ scheme ${ }^{22}$ fail to predict, even qualitatively, the trend of magnetism in this series of strongly correlated electron systems.

For the model Hamiltonian of the first method, ${ }^{4}$

$$
\begin{gathered}
H=\sum_{k} \epsilon_{k} c_{k}^{+} c_{k}+\sum_{R m} \epsilon_{m} f_{m}^{+}(R) f_{m}(R) \\
+\frac{U}{2} \sum_{R, m \neq m^{\prime}} n_{m}(R) n_{m^{\prime}}(R)+H_{1}, \\
H_{1}=\sum_{k m R}\left[V_{k m} e^{-\mathbf{i k} \cdot \mathbf{R}} c_{k}^{+} f_{m}(R)+\text { H.c. }\right] \\
-\sum_{k k^{\prime}} \sum_{m m^{\prime} R} J_{m m^{\prime}}\left(\mathbf{k}, \mathbf{k}^{\prime}\right) e^{-i\left(\mathbf{k}-\mathbf{k}^{\prime}\right) \mathbf{R}} c_{k}^{+} f_{m}^{+}(R) c_{k^{\prime}} f_{m^{\prime}}(R),
\end{gathered}
$$

the parameters involved, i.e., the band energies $\epsilon_{k}$, the $f$-state energy $\epsilon_{m}$, the on-site Coulomb repulsion $U$, the hybridization matrix elements $V_{k m}$, and the band- $f$ Coulomb exchange $J_{m m^{\prime}}\left(\mathbf{k}, \mathbf{k}^{\prime}\right)$, are evaluated on a wholly ab initio basis from nonspin polarized full potential linear muffin tin orbital $^{20}$ (FPLMTO) calculations. Note that the band- $f$ exchange interaction in $H_{1}$ is also included in the LDA treatment of the first two terms in Eq. (1). Thus, as in all approaches combining model Hamiltonians with density functional theory (for example, LDA $+U$ ), this ab initio based approach includes a certain amount of double counting of the band- $f$ exchange interaction. Our ab initio based approach is designed to correct for the situation that the treatment of the Coulomb interaction involving the rather localized $4 f$ electron is rather poor within LDA.

Because both the hybridization and Coulomb exchange matrix elements are much smaller $(\sim 0.1 \mathrm{eV})$ than the intra- atomic Coulomb interaction $U(6 \mathrm{eV})$, one can apply perturbative techniques and evaluate from first principles the anisotropic two-ion $6 \times 6$ interaction matrices $E_{m_{1} m_{1}^{\prime}}^{m_{2} m_{2}^{\prime}}(\mathbf{R})$, which couple the two $f$ ions. ${ }^{3,4}$ The $\mathrm{Ce}-\mathrm{Ce}$ interactions have three contributions: the wholly band- $f$ Coulomb exchange mediated interaction proportional to $J_{m m^{\prime}}^{2}$, the wholly hybridization-mediated exchange interaction proportional to $V_{k m}^{4}$, and the cross term proportional to $V_{k m}^{2} J_{m m^{\prime}}$. The lowtemperature magnetic moment and the ordering temperature can then be determined by use of a mean field calculation. ${ }^{3,4,7}$ We have previously ${ }^{3,4}$ applied this method to investigate the magnetic properties of the heavier (i.e., larger lattice constant) cerium systems $(\mathrm{CeBi}, \mathrm{CeSb}$, and $\mathrm{CeTe})$. However, these calculations did not take into account the CF interaction and employed a warped muffin-tin LMTO calculation for the parameters entering the model. The excellent agreement found ${ }^{4}$ with experiment for the magnetic moment and ordering temperature is relatively unaffected by the $\mathrm{CF}$ interaction, because the CF interaction in the heavier Ce systems is smaller than the two-ion interactions.

The second method employs ab initio electronic structure calculations, with the $4 f$ states treated as band states, based on the FPLMTO method ${ }^{20}$ using (1) only spin polarization, with the orbital polarization included only through the spinorbit coupling, (2) both spin and orbital polarization, ${ }^{21}$ and (3) the LDA $+U$ approach. $^{22}$

The crystalline field, neglected in the previous calculations, ${ }^{4,5}$ is expected to affect the magnetic behavior considerably, if it is large. The experimentally observed crystal-field splitting is $\Delta_{\mathrm{CF}}^{*}=\Delta_{\mathrm{CF}}+\delta \Delta_{\mathrm{CF}}$, where $\Delta_{\mathrm{CF}}$ is the "bare" CF splitting and $\delta \Delta_{\mathrm{CF}}$ is the hybridization-induced CF splitting. Hybridization between the band states and the $f$ states [Eq. (1) in Ref. 3] gives rise to a shift of the bare CF energy levels of the $4 f$ state multiplet. The bare CF energy of the $\left|\Gamma_{i}\right\rangle$ state can be evaluated in principle from FPLMTO calculations, namely, $E_{\Gamma i}=\left\langle\Gamma_{i}|V(\mathbf{r})| \Gamma_{i}\right\rangle$, where $V(\mathbf{r})$ is the $4 f$ muffin-tin potential expressed as a sum of lattice harmonics invariant under the $f$-ion point group operations, and the $f$ electrons are treated as localized rather than itinerant states. In the first method, we treat the $4 f$ states as core states, and allow them to interact only with the spherical component of the effective one-electron potential $V(\mathbf{r})$. Thus, both the hybridization-induced shift of the CF splitting and the interaction of the atomiclike $4 f$ state with the nonspherical components of the potential, giving rise to the CF splitting, are not included in the calculation of the model Hamiltonian parameters. Here, we generalize the first, $a b$ initio-based, method to include both the interatomic two-ion $4 f-4 f$ coupling and the $\mathrm{CF}$ interactions (one-ion term) on an equal footing and to employ a full potential LMTO evaluation of the model Hamiltonian parameters. While the effect of the full potential on both the hybridization and Coulomb exchange interactions is small, including the $\mathrm{CF}$ interaction will be shown to play a role as important as the interatomic $4 f-4 f$ interactions for understanding and predicting the overall trend in the unusual magnetic properties, as one chemically tunes the degree of $4 f$ localization. The resultant Hamiltonian is 
TABLE I. Calculated and experimental (Refs. 6-10) values of ordered magnetic moments for the cerium systems in $\mu_{B}$. Listed are the FLMTO values for the spin moment $\mu_{S}$, orbital moment $\mu_{L}$, and total moment $\mu$, for spin polarization (SP) only and for both spin and orbital polarization (OP) (Ref. 21).

\begin{tabular}{cccccccc}
\hline \hline & & $\mathrm{SP}$ & & & $\mathrm{SP}+\mathrm{OP}$ & & \multicolumn{2}{c}{ Exp. } \\
& $\mu_{S}$ & $\mu_{L}$ & $\mu$ & $\mu_{S}$ & $\mu_{L}$ & $\mu$ & $\mu$ \\
\hline $\mathrm{CeS}$ & -1.00 & 0.91 & -0.09 & -1.24 & 1.99 & 0.75 & 0.57 \\
$\mathrm{CeSe}$ & -1.08 & 1.02 & -0.06 & -1.26 & 2.07 & 0.81 & 0.57 \\
$\mathrm{CeTe}$ & -1.15 & 1.28 & 0.07 & -1.31 & 2.29 & 0.98 & 0.30 \\
& & & & & & & \\
$\mathrm{CeP}$ & -0.80 & 0.55 & -0.25 & -0.85 & 1.27 & 0.43 & 0.80 \\
$\mathrm{CeAs}$ & -0.84 & 0.64 & -0.20 & -0.85 & 1.42 & 0.57 & 0.80 \\
$\mathrm{CeSb}$ & -0.86 & 0.74 & -0.12 & -0.91 & 1.61 & 0.70 & 2.06 \\
$\mathrm{CeBi}$ & -0.86 & 0.74 & -0.12 & -0.95 & 1.69 & 0.74 & 2.10 \\
\hline \hline
\end{tabular}

$$
\begin{aligned}
H= & -\sum_{i, j} \sum_{\substack{\mu, \nu \\
\epsilon, \sigma}} \xi_{\mu \nu}^{\epsilon \sigma}\left(\theta_{i j}\right) e^{-i(\mu-\nu+\epsilon-\sigma) \phi_{i j}} \\
& \times c_{\epsilon}^{\dagger}(j) c_{\sigma}(j) c_{\mu}^{\dagger}(i) c_{\nu}(i)+B_{4} \sum_{i}\left[O_{4}^{0}(i)+5 O_{4}^{4}(i)\right],
\end{aligned}
$$

where the $\xi_{\mu \nu}^{\epsilon \sigma}\left(\theta_{i j}\right)$ are the two-ion interaction exchange matrices rotated to a common crystal-lattice axis, ${ }^{3}$ and the $O_{4}^{0}$ and $O_{4}^{4}$ are the Stevens operators equivalents acting on the $\mathrm{Ce}^{3+}$ free-ion $4 f_{5 / 2}$ multiplet. $^{23}$ The effective CF splitting is $\Delta_{\mathrm{CF}}^{*}=360 B_{4}$; a positive $B_{4}$ value gives the $\Gamma_{7}$ ground state, which is experimentally observed. While work in progress is currently aimed at evaluating the CF splitting on a wholly $a b$ initio basis, in the absence of an $a b$ initio value of the $\mathrm{CF}$ interaction in this class of strongly correlated cerium systems, $B_{4}$ is evaluated by setting $\Delta_{\mathrm{CF}}^{*}$ equal to the experimental values listed in Table III..$^{9,16}$

In Table I, we present the calculated values of the zerotemperature cerium magnetic moment from the FPLMTO electronic structure calculations assuming a ferromagnetic structure. Listed in the table are values both with and without the orbital polarization correction, beyond effects of spinorbit coupling, taken into account. Note, the importance of including the orbital polarization in these $4 f$ correlated electron systems. As expected, in all cases, the orbital polarization is found to be opposite to the spin polarization. Comparison of the total energies predicts that the magnetic anisotropy changes from the $\langle 001\rangle$ direction in the pnictides to the $\langle 111\rangle$ in the chalcogenides, in agreement with experiment. LDA calculations for $\mathrm{CeSb}$ for an $\mathrm{AF}-I$ structure give low spin and orbital moments of $-0.29 \mu_{B}$ and $0.43 \mu_{B}$, respectively, and an energy higher than the ferromagnetic state. Thus, except perhaps for the lighter chalcogenides ( $\mathrm{CeS}$ and $\mathrm{CeSe}$ ), comparison of the $a b$ initio and experimental values for the magnetic moment indicates the failure of the LDA calculations to treat properly the correlation effects of the $4 f$ states within the LDA as the degree of $4 f$ correlations increases in the heavier pnictide systems. LDA $+U$ calculations for the heavier systems $\mathrm{CeSb}$ and CeTe increase the magnetic moment to $2.2 \mu_{B}$ and $1.4 \mu_{B}$, respectively. ${ }^{22}$ Similarly, we find that the LDA $+U$ method increases the magnetic moment for the lighter, more delocalized systems, $\mathrm{CeP}$ and $\mathrm{CeS}$ to $2.0 \mu_{B}$ and $1.9 \mu_{B}$, respectively. Thus, the $\mathrm{LDA}+U$ method enhances the magnetic moment across the entire series and fails to predict the trend of magnetic properties. Furthermore, all $a b$ initio calculations fail to predict the moment collapse from $\mathrm{CeSb}$ to $\mathrm{CeTe}$, the latter being an incipient heavy Fermion system. ${ }^{18}$

In Table II, we list the values of the $m=m^{\prime}=1 / 2$ matrix elements (characteristic matrix elements of the $6 \times 6$ exchange interaction matrix) for the first three nearest-neighbor shells for the light ( $\mathrm{CeP}$ and $\mathrm{CeS}$ ) and the heavier compounds (CeSb and $\mathrm{CeTe}$ ). Listed separately are the three contributions to the interatomic $4 f-4 f$ interactions arising from band- $f$ hybridization $\left(V^{4}\right)$, band- $f$ Coulomb exchange $\left(J^{2}\right)$, and the cross term. It is important to note that while the Coulomb exchange mediated interactions overall dominate the magnetic behavior for the heavier, more localized, $4 f$ systems, the opposite is true for the lighter, more delocalized, systems where (in the absence of CF effect) the hybridization mediated interactions dominate the magnetic behavior. This change of behavior of the interatomic $4 f-4 f$ interactions is a result of the sensitivity of the hybridization and Coulomb exchange to the degree of $4 f$ localization. Equally important, is that while both first and second nearest-neighbor $4 f-4 f$ interactions are ferromagnetic (FM) for $\mathrm{CeSb}$, there is an interplay between ferromagnetic first nearest-neighbor and antiferromagnetic (AF) second nearestneighbor interactions for CeTe. This results in a saturated moment for $\mathrm{CeSb}$ and in the ordered magnetic moment collapse for CeTe (see Table III).

To determine whether the magnetic moment collapse might be of Kondo origin, we have evaluated the single-impurity Kondo temperature $k_{B} T_{K}$ $=D(D / \xi)^{4 / 3}\left(D / \Delta_{\mathrm{CF}}^{*}\right)^{2} e^{-1 / 2 \rho\left(E_{F}\right)\left|J\left(E_{F}\right)\right|}$, across the series, for the case of a $\Gamma_{7}$ ground state. ${ }^{24}$ Here, $D$ is the conductionelectron bandwidth, $\rho\left(E_{F}\right)$ is the density of states of the conduction electrons at the Fermi energy $E_{F}, \xi$ is the spinorbit coupling, and $J\left(E_{F}\right)$ is the conduction electron- $f$ effective exchange interaction at $E_{F}$, which has contributions both from the Coulomb exchange interaction in Eq. (2), provided that it is negative, and the hybridization-induced interaction $J_{\text {hyb }}\left(E_{F}\right)=-V^{2}\left(E_{F}\right) U /\left|E_{f}-E_{F}\right|\left(\left|E_{f}-E_{F}\right|+U\right)$. The Coulomb exchange interaction at $E_{F}$ is positive across the 
TABLE II. Values of the $m=m^{\prime}=1 / 2$ matrix elements of the $6 \times 6$ interatomic $4 f-4 f$ interaction matrix $E_{m_{a} m_{a}^{\prime}}^{m_{b} m_{b}^{\prime}}(\mathbf{R})$, for the first three nearest-neighbor shells in $\mathrm{K}$. We list also values of the hybridization induced $\left(E_{V^{4}}\right)$, cross term $\left(E_{V^{2} J}\right)$, and Coulomb exchange $\left(E_{J^{2}}\right)$ contributions.

\begin{tabular}{|c|c|c|c|c|c|c|}
\hline & \multicolumn{3}{|c|}{$\mathrm{CeP}$} & \multicolumn{3}{|c|}{$\mathrm{CeS}$} \\
\hline & $E_{V^{4}}$ & $E_{V^{2} J}$ & $E_{J^{2}}$ & $E_{V^{4}}$ & $E_{V^{2} J}$ & $E_{J^{2}}$ \\
\hline$R=\left(\frac{1}{2} \frac{1}{2} 0\right)$ & 2.23 & 0.64 & 1.53 & 0.85 & -0.40 & 1.50 \\
\hline$R=\left(\begin{array}{lll}1 & 0 & 0\end{array}\right)$ & 6.39 & 0.27 & 1.65 & -1.60 & 0.04 & -0.80 \\
\hline \multirow{3}{*}{$R=\left(1 \frac{1}{2} \frac{1}{2}\right)$} & -0.08 & -0.02 & 0.16 & 0.38 & -0.16 & 0.13 \\
\hline & \multicolumn{3}{|c|}{$\mathrm{CeSb}$} & \multicolumn{3}{|c|}{$\mathrm{CeTe}$} \\
\hline & $E_{V^{4}}$ & $E_{V^{2} J}$ & $E_{J^{2}}$ & $E_{V^{4}}$ & $E_{V^{2} J}$ & $E_{J^{2}}$ \\
\hline$R=\left(\frac{1}{2} \frac{1}{2} 0\right)$ & 0.70 & 0.34 & 7.30 & 0.17 & -0.19 & 2.90 \\
\hline$R=\left(\begin{array}{lll}1 & 0 & 0\end{array}\right)$ & 2.07 & 0.07 & 10.21 & -0.19 & 0.04 & -1.69 \\
\hline$R=\left(1 \frac{1}{2} \frac{1}{2}\right)$ & -0.02 & -0.03 & 0.40 & 0.04 & -0.06 & -0.01 \\
\hline
\end{tabular}

entire series and hence cannot give rise to the Kondo effect. Thus, only $J_{\text {hyb }}\left(E_{F}\right)$, can give rise to the Kondo effect. ${ }^{24}$ Using the ab initio values of the parameters entering the expression for $T_{K}$, we find that $T_{K} \ll T_{\text {ord }}$ across the series $\left(T_{K}<10^{-4} \mathrm{~K}\right)$. These results show that the moment collapse from $\mathrm{CeSb}$ to $\mathrm{CeTe}$ is of non-Kondo origin. Rather, it results from an interplay of $\mathrm{FM}$ and $\mathrm{AF}$ interatomic $4 f-4 f$ interactions which arises purely from differences in the underlying electronic structure.

Listed in Table III are the calculated zero-temperature ordered moment and ordering temperature $T_{N}$ from the first, $a b$ initio-based, method, with and without the $\mathrm{CF}$ interaction. It is clear that for the heavier systems $(\mathrm{CeBi}, \mathrm{CeSb}, \mathrm{CeTe})$ the effect of the CF interaction on the magnetic moments is small, and it is slightly more pronounced on the ordering temperatures. This is due to the fact that for the more localized systems the CF interaction is smaller than the two-ion interactions. This is the reason that the previous calculations, ${ }^{4}$ neglecting the $\mathrm{CF}$ interaction, gave results in excellent agreement with experiment. On the other hand, for the lighter more delocalized systems the $\mathrm{CF}$ interactions are much larger than the interatomic $4 f-4 f$ interactions, and hence dominate the magnetic behavior. Overall, the first, $a b$ initio-based, approach which takes into account all three pertinent interactions (hybridization, Coulomb exchange, and CF interactions) on an equal footing, yields results for both the zero-temperature moment and the ordered temperature (a more stringent test for the theory) in excellent agreement with experiment.

A final corroboration of the success of the first ab initiobased method is that it predicts the experimentally observed change of the ground-state magnetic structure from the $\langle 001\rangle$ $\mathrm{AF}-I A$ in $\mathrm{CeBi}$ and $\mathrm{CeSb}$ to the $\langle 001\rangle \mathrm{AF}-I$ in $\mathrm{CeAs}$ and $\mathrm{CeP}^{6,14}$ The sign (FM or AF) of the $| \pm 5 / 2\rangle$ matrix elements of the $6 \times 6$ exchange matrix determines the interplanar interaction between successive (001) Ce planes. We find, that for the heavier compounds (CeBi and $\mathrm{CeSb}$ ) the $| \pm 5 / 2\rangle$ matrix elements of the Coulomb exchange matrix are FM and hence favor the $\uparrow \uparrow \downarrow \downarrow$ type, while in the lighter systems (CeAs and $\mathrm{CeP}$ ) the $| \pm 5 / 2\rangle$ matrix elements of the hybridization-induced two-ion matrix are $\mathrm{AF}$, and hence they favor the $\uparrow \downarrow$ type.

In conclusion, we have applied two different, viz., $a b$ initio-based and ab initio LDA methods to study the dramatic change of magnetic properties across a series of strongly correlated electron systems which offer the oppor-

TABLE III. Calculated (from the first, ab initio based, method) and experimental (Refs. 6-10) values of the zero-temperature ordered moment $\mu$ and the ordering temperature $T_{N}$ with and without the CF interaction across the series. Also listed are the experimental values (Refs. 9 and 16) of the CF splitting $\Delta_{\mathrm{CF}}^{*}$.

\begin{tabular}{|c|c|c|c|c|c|c|c|}
\hline & \multirow[b]{2}{*}{$\Delta_{\mathrm{CF}}^{*}(\mathrm{~K})$} & \multicolumn{3}{|c|}{$\mu\left(\mu_{B}\right)$} & \multicolumn{3}{|c|}{$T_{N}(\mathrm{~K})$} \\
\hline & & no $\mathrm{CF}$ & $\mathrm{CF}$ & exp. & no $\mathrm{CF}$ & $\mathrm{CF}$ & exp. \\
\hline $\mathrm{CeS}$ & 140 & 1.80 & 0.73 & 0.57 & 1.0 & 11.0 & 8.4 \\
\hline $\mathrm{CeSe}$ & 116 & 1.10 & 0.79 & 0.57 & 2.5 & 14.0 & 5.7 \\
\hline $\mathrm{CeTe}$ & 32 & 0.60 & 0.46 & 0.30 & 8.0 & 5.0 & 2.2 \\
\hline $\mathrm{CeP}$ & 150 & 2.10 & 0.73 & 0.81 & 14 & 11 & 8 \\
\hline CeAs & 137 & 2.10 & 0.74 & 0.85 & 16 & 13 & 8 \\
\hline $\mathrm{CeSb}$ & 37 & 2.10 & 1.80 & 2.06 & 20 & 18 & 17 \\
\hline $\mathrm{CeBi}$ & 8 & 2.10 & 2.10 & 2.10 & 40 & 40 & 26 \\
\hline
\end{tabular}


tunity to chemically tailor the different type of interactions (band- $f$ hybridization, band $f$ Coulomb exchange, and CF interactions), pertinent to the magnetic behavior. The first $a b$ initio-based approach which explicitly takes into account the interplay of the three pertinent interactions, gives results in excellent agreement with experiment for all compounds in the series, including the moment collapse from $\mathrm{CeSb}$ to $\mathrm{CeTe}$ and the trend of moments and ordering temperatures across the series. On the other hand, the second, $a b$ initio LDA, method gives good results for the lighter chalcogenides, but it entirely fails to give, even qualitatively, the trend of the unusual magnetic behavior.

The research at CSUN was supported by NSF Grant No. DMR-9531005 and U.S. Army Grant No. DAAH04-95, and at WVU by NSF Grant No. DMR-9120333.
${ }^{1}$ Correlation Effects and Materials Properties, edited by A. Gonis, N. Kioussis, and M. Ciftan (Kluwer Academic/Plenum, New York, 1999).

${ }^{2}$ D. W. Hess, P. S. Riseborough, and J. L. Smith, Heavy Fermion Phenomena, Encyclopedia of Applied Physics, edited by G. Trigg (VCH, New York, 1993), Vol. 71, p. 435.

${ }^{3}$ N. Kioussis, B. R. Cooper, and J. M. Wills, Phys. Rev. B 44, 10003 (1991).

${ }^{4}$ Q. G. Sheng and B. R. Cooper, J. Appl. Phys. 69, 5472 (1991); Phys. Rev. B 50, 965 (1994).

${ }^{5}$ B. R. Cooper, O. Vogt, Q. G. Sheng, and Y. L. Lin, Philos. Mag. B 79, 683 (1999).

${ }^{6}$ J. Rossat-Mignod, P. Burlet, S. Quezel, J. M. Effantin, D. Delacôte, H. Bartholin, O. Vogt, and D. Ravot, J. Magn. Magn. Mater. 31-34, 398 (1983).

${ }^{7}$ B. R. Cooper, R. Siemann, D. Yang, P. Thayamballi, and A. Banerjea, in Handbook of the Physics and Chemistry of the Actinides, edited by A. J. Freeman and G. H. Lander (NorthHolland, Amsterdam, 1985), Vol. 2, p. 435.

${ }^{8}$ A. Dönni, A. Furrer, P. Fischer, and F. Hulliger, Physica B 186188, 541 (1993).

${ }^{9}$ F. Hulliger, B. Natterer, and H. R. Ott, J. Magn. Magn. Mater. 8, 87 (1978).

${ }^{10}$ H. R. Ott, J. K. Kjems, and F. Hulliger, Phys. Rev. Lett. 42, 1378 (1979)

${ }^{11}$ J. Rossat-Mignod, J. M. Effantin, P. Burlet, T. Chattopadhyay, L.
P. Regnault, H. Bartholin, C. Vettier, O. Vogt, D. Ravot, and J. C. Achart, J. Magn. Magn. Mater. 52, 111 (1985).

${ }^{12}$ N. Môri, Y. Okayama, H. Takahashi, Y. Haga, and T. Suzuki, Physica B 186-188, 444 (1993).

${ }^{13}$ S. Doniach, Physica B 91, 231 (1977).

${ }^{14}$ Y. Okayama, Y. Ohara, S. Mituda, H. Takahashi, H. Yoshizawa, T. Osakabe, M. Kohgi, Y. Haga, T. Suzuki, and N. Môri, Physica B 186-188, 531 (1993).

${ }^{15}$ R. J. Birgeneau, E. Bucher, J. P. Maita, L. Passell, and K. C. Turberfield, Phys. Rev. B 8, 5345 (1973).

${ }^{16}$ J. Rossat-Mignod, J. M. Effantin, P. Burlet, T. Chattopadhyay, L. P. Regnault, H. Bartholin, C. Vettier, O. Vogt, D. Ravot, and J. C. Achart, J. Magn. Magn. Mater. 52, 111 (1985).

${ }^{17}$ J. M. Wills and B. R. Cooper, Phys. Rev. B 36, 3809 (1987).

${ }^{18}$ H. R. Ott (private communication), as cited in B. R. Cooper, Q. G. Sheng, S. P. Lim, C. Sanchez-Castro, N. Kioussis, and J. M. Wills, J. Magn. Magn. Mater. 108, 10 (1992).

${ }^{19}$ P. Santini, R. Lemanski, and P. Erdos, Adv. Phys. 48, 537 (1999).

${ }^{20}$ D. L. Price and B. R. Cooper, Phys. Rev. B 39, 4945 (1989).

${ }^{21}$ M. S. Brooks and P. J. Kelly, Phys. Rev. Lett. 51, 1708 (1983).

${ }^{22}$ D. Price, B. R. Cooper, S. P. Lim, and I. Avgin, Phys. Rev. B 61, 9867 (2000).

${ }^{23}$ K. R. Lea, M. J. M. Leask, and W. P. Wolf, J. Phys. Chem. Solids 23, 1381 (1962).

${ }^{24}$ K. Yamada, K. Yosida, and K. Hanzawa, Prog. Theor. Phys. 71, 450 (1984) 